\title{
TOMOGRAFIA COMPUTADORIZADA NA AGENESIA DO CORPO CALOSO: ACHADOS EM 27 CASOS
}

\author{
GUILBERTO MINGUETTI*, KARINE FURTADO** LISANDRA CARLA DE AGOSTINI**
}

\begin{abstract}
RESUMO - Foi realizado estudo retrospectivo de tomografia computadorizada em 27 pacientes com anormalidades anatômicas definidas como agenesia total do corpo caloso, afastando-se os casos de agenesia parcial. Cada caso foi avaliado buscando-se as características tomográficas, incidência por sexo, idade, associação com a clínica e com outras malformações congênitas encefálicas. Nesta casuística, contudo, foram eliminados os casos de agenesia do corpo caloso associados a esquizencefalias, holoprosencefalias e malformações do complexo de Dandy-Walker.
\end{abstract}

PALAVRAS-CHAVE: corpo caloso, agenesia, tomografia computadorizada.

\section{Computerized tomography in the agenesis of the corpus callosum: a description of 27 cases}

ABSTRACT - We describe a retrospective study of 27 cases of complete agenesis of the corpus callosum examined by $\mathrm{CT}$ and not associated to schizencephaly, holoprosencephaly and Dandy-Walker complex. Partial agenesis is also not included in the present study. The imaging findings are correlated to sex, age and symptoms.

KEY WORDS: corpus callosum, agensis, CT scan.

O corpo caloso é a maior via de associação entre os hemisférios cerebrais. É formado por grande número de fibras que cruzam o plano sagital mediano e penetram de cada lado do cérebro unindo áreas simétricas do córtex cerebral de cada hemisfério ${ }^{1}$. Sua formação se inicia em torno da $12^{\mathrm{a}}$ semana de gestação e ele encontra-se completamente desenvolvido entre a $18^{\mathrm{a}}$ e $20^{\mathrm{a}}$ semanas de vida intra-uterina ${ }^{2,3}$.

Sua função é permitir a transferência de informações entre um hemisfério e outro fazendo com que eles atuem harmonicamente ${ }^{1}$. A ausência do corpo caloso pode ser total ou parcial. Estas alterações são encontradas em 1 a $3: 1000$ crianças nascidas vivas ${ }^{4,5}$. Embora seja mais comum a malformação isolada, ela é encontrada em associação com cerca de 25 síndromes genéticas, erros inatos do metabolismo e em associação com o uso abusivo de álcool e cocaína pela mãe $e^{6,7}$. Pode ocorrer como lesão isolada ou como parte de outras anomalias craniocerebrais, como o complexo de Dandy-Walker, polimicrogiria, heterotopias, lipoma, encefalocele, cisto aracnoídeo, esquizencefalia, holoprosencefalia e outras ${ }^{5,8,9}$. Agenesia do corpo caloso pode ser assintomática, mas epilepsia, atraso do desenvolvimento neuropsicomotor (DNPM) e outras manifestações clínicas são achados comuns, bem como alterações eletrencefalográficas ${ }^{10,11,12}$.

O objetivo deste estudo é descrever os achados tomográficos em 27 casos de agenesia do corpo caloso.

Disciplina de Neurologia do Departamento de Clínica Médica da Universidade Federal do Paraná (UFPR) e CETAC - Centro de Tomografia Computadorizada, Curitiba, Paraná: *Professor Adjunto; **Sextanista do Curso de Medicina. Aceite: 29-julho-1998.

Dr. Guilberto Minguetti - Rua Padre Ildefonso 105 - 80240-160 Curitiba PR - Brasil. 
Tabela 1. Grupo etário dos pacientes.

\begin{tabular}{cc}
\hline Idade (anos) & $\mathrm{N}^{\mathrm{o}}$ de pacientes \\
\hline $0-1$ & 15 \\
$2-5$ & 5 \\
$6-10$ & 2 \\
$11-15$ & 2 \\
$16-20$ & 1 \\
$21-25$ & 1 \\
$26-30$ & 0 \\
$31-35$ & 1 \\
\hline
\end{tabular}

\section{CASUÍSTICA E MÉTODO}

Foram selecionados para o presente estudo 27 pacientes com o diagnóstico de agenesia total do corpo caloso pela tomografia computadorizada, no período de 1978 a 1997. O estudo foi realizado no CETAC, em Curitiba, tendo sido utilizados os aparelhos Delta Scan 25 (Ohio-Nuclear) com cortes tomográficos axiais de 5,8 e $10 \mathrm{~mm}$ de espessura e Tomoscan 350 (Philips) com cortes tomográficos de 6 e $9 \mathrm{~mm}$ de espessura. Nos dois aparelhos a matriz utilizada foi 256x256. Cada caso foi avaliado segundo a idade do paciente na época da realização do exame, sexo, manifestações clínicas e associação com outras malformações cerebrais avaliadas no mesmo exame tomográfico.
Tabela 2. Manifestações clínicas em 20 pacientes analisados.

\begin{tabular}{cc}
\hline Manifestações clínicas & $N^{o}$ de pacientes \\
\hline Atraso do DNPM & 9 \\
Crises convulsivas & 9 \\
Hemiparesia & 2 \\
Cefaléia & 3 \\
Hipotonia & 1 \\
Outros & 3 \\
\hline
\end{tabular}

DNPM, desenvolvimento neurpsicomotor.

Tabela 3. Malformações encefálicas associadas em 8 pacientes.

\begin{tabular}{cc}
\hline Malformações & $N^{o}$ de pacientes \\
\hline Cistos inter-hemisféricos & 2 \\
Atrofia cerebral & 2 \\
Microcefalia & 1 \\
Leucomalácia ventricular & 1 \\
Atrofia cerebelar & 1 \\
Encefalocele occipital & 1 \\
\hline
\end{tabular}

\section{RESULTADOS}

Dos 27 pacientes com diagnóstico tomográfico de agenesia total de corpo caloso, 16 eram do sexo masculino (59\%) e 11 do sexo feminino (41\%). A idade na época da realização do exame variou de 23 dias a 33 anos, segundo a distribuição na Tabela 1.

Tivemos acesso às manifestações clínicas dos pacientes por meio de requisições de exames em apenas 20 casos, segundo a distribuição na Tabela 2.

A associação com outras malformações encefálicas foi observada em 8 pacientes $(29,5 \%)$; elas estão relacionadas na Tabela 3.

Os principais achados tomográficos (Fig 1) encontrados pela ordem de frequência foram: 1 separação dos ventrículos laterais com seus corpos paralelos e ausência de septo pelúcido; 2 - alteração da forma dos ventrículos laterais; 3 - dilatação dos cornos occipitais dos ventrículos laterais (colpocefalia); 4 - fissura inter-hemisférica estendendo-se até a porção anterior do terceiro ventrículo; 5 - assimetria dos ventrículos laterais; 6 - afilamento dos cornos frontais dos ventrículos laterais; 7 - cistos inter-hemisféricos; 8 - coleção fluida extracerebral.

\section{DISCUSSÃO}

Agenesia de corpo caloso é anomalia encefálica congênita incomum que acomete com igual frequência pacientes do sexo masculino e feminino. Em nosso estudo, houve predominância discreta do sexo masculino. O desenvolvimento embrionário do corpo caloso se dá entre a $12^{\mathrm{a}}$ e $20^{\mathrm{a}}$ semanas de gestação, concomitantemente com o desenvolvimento de muitas outras estruturas encefálicas ${ }^{8,9,13,14}$. As anomalias congênitas encefálicas resultam de insulto (vascular, metabólico, infeccioso, ou outros) 

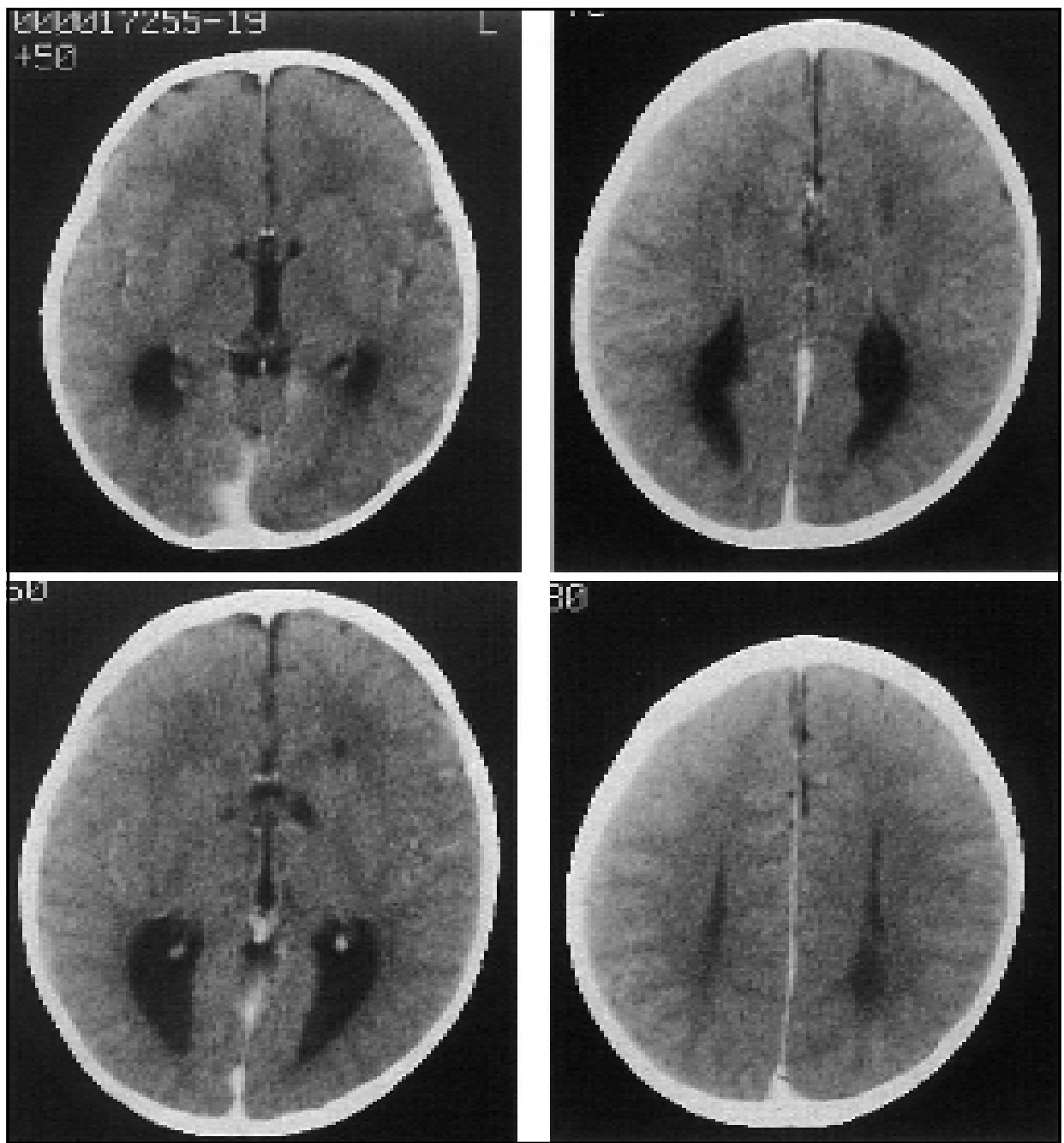

Fig 1. Cortes axiais de $5 \mathrm{~mm}$ de espessura da base para o ápice, após a injeção endovenosa do contraste iodado, mostram a separação dos ventrículos laterais cujos corpos mantêm-se paralelos entre si. Notar a ausência do septo pelúcido. Observar a impossibilidade de se identificar os cornos anteriores e a dilatação dos cornos posteriores dos ventrículos laterais. Não são observados acúmulos patológicos do contraste.

durante o período de desenvolvimento cerebral, dependente do tempo de exposição e à susceptibilidade genética do embrião ou feto ao insulto. O insulto, se localizado, pode determinar a lesão de corpo caloso isoladamente. Se o insulto for difuso, muitas das estruturas encefálicas que se desenvolvem concomitantemente podem estar afetadas e esta é a condição mais comum ${ }^{13,15}$. A associação com outras malformações cerebrais foi encontrada apenas em $29,5 \%$ dos nossos pacientes, talvez explicada por termos excluído os casos de agenesia parcial de corpo caloso e os casos associados às esquizencefalias, holoprosencefalias e ao complexo de Dandy-Walker.

O significado clínico da agenesia isolada do corpo caloso é de menor importância, porque sua ausência é compensada por conexões não-calosas, desenvolvimento bilateral de funções específicas e pela utilização de vias sensoriais somáticas ipsilaterais ${ }^{11,16}$. A condição é usualmente identificada incidentalmente durante a investigação de outras anomalias e são estas que, quando presentes, darão 
os sintomas de epilepsia, problemas neurológicos e atraso do desenvolvimento ${ }^{16,10}$. A idade do diagnóstico em nossos pacientes variou de 23 dias a 33 anos, demonstrando a variabilidade das manifestações clínicas e suas intensidades. A relação entre sintomatologia e malformações associadas não pôde ser realizada, pois não tínhamos o quadro clínico de todos os pacientes.

Quando o corpo caloso está ausente, os axônios que iriam cruzar o plano mediano retornam à fissura inter-hemisférica e correm paralelamente a esta formando os feixes calosos longitudinais de Probst $^{14}$. Devido a sua localização, os feixes de Probst dão aos ventrículos laterais uma forma crescente mais pronunciada rostralmente. O terceiro ventrículo tende a ser mais largo e estender-se superiormente dentro da fissura inter-hemisférica formando uma coleção líquida, geralmente referida como cisto inter-hemisférico. O forame de Monro tende a alargar-se. É o corpo caloso que dá ao ventrículo lateral sua forma e mantém seu tamanho normal. Na ausência de corpo caloso os ventrículos laterais mantêm-se paralelos e suas paredes mediais apresentam-se côncavas. Os cornos occipitais podem mostrar relativa dilatação. Este fenômeno chama-se colpocefalia e deve-se à ausência do esplênio do corpo caloso.

Os achados tomográficos da agenesia de corpo caloso são bem caracterizados e bem definidos em cortes axiais e coronais ${ }^{6}$. Podemos observar nas imagens tomográficas a separação dos ventrículos laterais, seus corpos paralelos, a alteração de suas formas, ausência de septo pelúcido, colpocefalia, coleção fluida ou cistos inter-hemisféricos, extensão da fissura inter-hemisférica até a porção anterior do terceiro ventrículo, afilamento dos cornos frontais dos ventrículos laterais, supra-elevação e interposição do terceiro ventrículo ao corpo do ventrículo lateral, aspecto cístico ou dilatação do terceiro ventrículo e as alterações tomográficas específicas para cada malformação encefálica associada ${ }^{17}$. Estes dados da literatura coincidem com os achados em nosso estudo.

\section{CONCLUSÃO}

Embora a ressonância magnética seja o melhor método diagnóstico para as diferentes disgenesias do corpo caloso, a tomografia computadorizada mostra-se método confiável nos casos específicos de agenesia total do corpo caloso. Procuramos no presente estudo fazer um levantamento estatístico destes casos, encontrando 25 casos em 20 anos de atividade clínica e mostramos que as alterações observadas são típicas e coincidentes com outros estudos anteriormente registrados.

\section{REFERÊNCIAS}

1. Machado A. Anatomia funcional. 2.Ed. Rio de Janeiro: Ateneu, 1993.

2. Moore KL. Embriologia clínica. 4.Ed. Rio de Janeiro: Guanabara Koogan, 1990.

3. Rakic P, Yakovlev PI. Development of the corpus callosum and cavum septi in man. J Comp Neurol 1968;132:45-72.

4. Jeret JS, Serun C, Wiesniewski K, Fish C. Frequency of agenesis of the corpus callosum in the developmentally disable population as determined by computerized tomography. Pediatric Neurosci 1986;12:101-103.

5. Ford FR. Diseases of the nervous system in infancy, childhood and adolescence. 5.Ed. Springfield: Charles C. Thomas, 1966.

6. Kolodny EH. Agenesis of the corpus callosum: a marker for inherited metabolic disease? Neurology 1989;39:847-848.

7. Wiesniewski K, Damska M, Sher JH, Oazi O. A clinical neuropathological study of the fetal alcohol syndrome. Neuropediatrics 1983;14:197-201.

8. Barkovich AJ, Norman D. Anomalies of the corpus callosum. In: Vinken PJ, Bruyn GW. (eds). Handbook of clinical neurology, Vol 30. Amsterdam: North Holland, 1977: 285-297.

9. Parrish MD, Roessmann U, Levinsohn MH. Agenesis of the corpus callosum: a study of the frequency of associated malformations. Ann Neurol 1979;6:349-356.

10. Byrd SE, Harwood-Nash DC, Fitz CR. Absence of the corpus callosum: computed tomografic evaluation in infant and children. J Can Assoc Radiol 1978;29:108-112.

11. Guilbert-Trainer F, Piton J, Billerey J, Caille JM. Agenesis of corpus callosum. J Neuroradiol 1982;9:135-160.

12. Grogno JL. Children with agenesis of the corpus callosum. Dev Med Child Neurol 1968;10:613-616.

13. Barkovich AJ, Norman D. Anomalies of the corpus callosum: correlation with further anomalies of the brain. Am J Roent 1988;151:171-179.

14. Kendall BE. Disgenesis of the corpus callosum. Neuroradiology 1983;25:239-256.

15. Kemeyama Y. Comparative developmental pathology of malformations of the central nervous system. Progr Clin Biol Res 1985; 163A:143-156.

16. Warren ME, Cook JV. Agenesis of the corpus callosum: case report. Br J Radiol 1993; 66:81-85.

17. Guiorsy M, Jaccone A, Fondelli MP, Salomone G. TC e disgenesia del corpo caloso. Min Ped 1988;40:599-604.

18. Probst FP. Congenital defects of the corpus callosum: morphology and encephalographic appearances. Acta Radiol 1973;331:1-152. 